\title{
Chinese Herbal Medicine for Infertility with Anovulation: A Systematic Review
}

\author{
Li Tan, MMed, ${ }^{1,2}$ Yao Tong, PhD, ${ }^{2}$ Stephen Cho Wing Sze, PhD, ${ }^{2}$ Mei Xu, MMed(cand), \\ Yang Shi, MMed(cand), Xin-yang Song, MMed, ${ }^{3}$ and Ting-ting Zhang, $\mathrm{PhD}^{1}$
}

\begin{abstract}
The aim of this systematic review is to assess the effectiveness and safety of Chinese herbal medicine (CHM) in treatment of anovulation and infertility in women. Eight (8) databases were extensively retrieved. The Chinese electronic databases included VIP Information, CMCC, and CNKI. The English electronic databases included AMED, CINAHL, Cochrane Library, Embase, and MEDLINE ${ }^{\circledR}$. Randomized controlled trials using CHM as intervention were included in the study selection. The quality of studies was assessed by the Jadad scale and the criteria referred to Cochrane reviewers' handbook. The efficacy of CHM treatment for infertility with anovulation was evaluated by meta-analysis. There were 692 articles retrieved according to the search strategy, and 1659 participants were involved in the 15 studies that satisfied the selection criteria. All the included trials were done in China. Meta-analysis indicated that CHM significantly increased the pregnancy rate (odds ratio [OR] $3.12,95 \%$ confidence interval [CI] 2.50-3.88) and reduced the miscarriage rate (OR $0.2,95 \%$ CI $0.10-0.41$ ) compared to clomiphene. In addition, CHM also increased the ovulation rate (OR 1.55, 95\% CI 1.06-2.25) and improved the cervical mucus score (OR 3.82, 95\% CI 1.78-8.21) compared to clomiphene, while there were no significant difference between $\mathrm{CHM}$ and clomiphene combined with other medicine. CHM is effective in treating infertility with anovulation. Also, no significant adverse effects were identified for the use of CHM from the studies included in this review. However, owing to the low quality of the studies investigated, more randomized controlled trials are needed before evidence-based recommendation regarding the effectiveness and safety of $\mathrm{CHM}$ in the management of infertility with anovulation can be provided.
\end{abstract}

\section{Introduction}

I NFERTILITY IS DEFINED as failure to achieve pregnancy during 1 year of frequent, unprotected intercourse. ${ }^{1}$ Infertility affects about $10 \%-20 \%$ of couples trying to achieve pregnancy in many industrialized countries, ${ }^{2}$ and there is an increasing number of couples seeking medical treatment. ${ }^{3} \mathrm{~A}$ study estimates that in 2002, more than 186 million evermarried women of reproductive age in developing countries were infertile. ${ }^{4}$

It is known that about $40 \%$ of infertility cases are due to female factors. Ovulatory dysfunction will be identified in approximately $15 \%$ of all infertile couples and accounts for up to $40 \%$ of infertility in women..$^{5}$ Ovulatory dysfunction is defined as abnormal, irregular, or absent ovulation. Approximately $15 \%--25 \%$ of patients with ovulatory dysfunction are anovulatory, ${ }^{6}$ which can be at- tributed to an imbalance of luteinizing hormone and follicle-stimulating hormone, an injury to the hypothalamus or pituitary gland, pituitary tumors, or too low or too high a body weight. ${ }^{7}$

Methods for evaluating ovulatory function may include: menstrual history, basal body temperature (BBT), serum progesterone, urinary luteinizing hormone, endometrial biopsy, histologic evaluation, and serial transvaginal ultrasound. ${ }^{8-11}$

Hull reported that the most successful treatment is for women with clearly defined ovulatory disorders. ${ }^{12}$ In most women with ovulatory dysfunction without an evident cause or that is not otherwise correctable, ovarian stimulation is the primary treatment. ${ }^{13}$ Fertility drugs are often used to induce ovulation. If they fail as the sole therapy, they may be used with assisted reproductive procedures, such as in vitro fertilization. ${ }^{14}$

\footnotetext{
${ }^{1}$ YueYang Hospital of Integrative Chinese \& Western Medicine Affiliated with Shanghai University of Traditional Chinese Medicine, Shang Hai, China.

${ }^{2}$ School of Chinese Medicine, LKS Faculty of Medicine, The University of Hong Kong, Hong Kong SAR, China.

${ }^{3}$ Shanghai University of Traditional Chinese Medicine, Shang Hai, China.
} 
Clomiphene citrate has been used as the first-line treatment for inducing ovulation in anovulatory women. ${ }^{15,16}$ In most women who fail to ovulate or conceive with clomiphene citrate, gonadotrophins bring about ovulation. ${ }^{17,18}$ Recent studies have reported that the aromatase inhibitor, letrozole, is an attractive option. ${ }^{19}$ Because the cloud of imputed teratogenesis is difficult to dispel with finality, aromatase inhibitors at present remain second-line agents. ${ }^{20}$

Ovulation-inducting agents have their own specific adverse effects. Some, in particular those effects associated with gonadotrophins, may be life threatening. ${ }^{21}$ In addition, induction of ovulation has been shown to raise the risk of miscarriage. ${ }^{22}$ With regard to the long-term safety of these medications, the relationship between fertility drugs and epithelial ovarian cancer is controversial. ${ }^{23-29}$ Assisted reproductive technology is highly effective when it comes to increasing conception, but most people cannot afford the cost. It is often associated with many physical and emotional side-effects, such as ovarian hyperstimulation syndrome (OHSS), multiple births, and birth defects. ${ }^{30-33}$

For this reason, more couples are turning to alternative medicine, especially Chinese herbal medicine $(\mathrm{CHM}){ }^{34-36}$ The use of herbal medicinal products is rapidly increasing. Virtually all survey data agree that users of herbal medicine products are predominantly female. ${ }^{37-39}$

Traditional Chinese medicine (TCM) is the world's oldest continuous surviving tradition, a 3000-year-old holistic system for both treatment and prevention of disease. Records indicate that Chinese medicinal herbs have a long history of use in treating male and female infertility for more than 2000 years. Chinese medicine practitioners believe that anovulation is mostly caused by deficiency of Kidney, Stasis of Liver Qi (keep in mind that in Chinese Medicine, there is more to an "organ" than its Western anatomical counterpart), Phlegm Dampness, and Blood Stasis (they are a syndrome identified by Chinese Medicine). ${ }^{40-42}$ In these cases, the aims of TCM therapy are tonifying and replenishing Kidney $Q i$, enriching and tonifying Kidney $Y$ in, warming and tonifying Kidney Yang, soothing the Liver and releasing depression, drying Dampness, and resolving Phlegm, activating Blood, and resolving Stasis. ${ }^{41-43}$

Herbs such as Radix rehmanniae, Radix Dipsaci, semen cuscutae, Cistanches, Fructus Lycii, Radix Morindae Officinalis, Epimedii, Cornu Cervi Degelatinatum, and Placenta Hominis are used to tonify the Kidney; Radix Bupleuri, Radix Paeoniae Alba, Fructus Aurantii, Rhizoma Cyperi, and Radix Curcumae are used to sooth the Liver and release depression; Rhizoma Atractylodis, Rhizoma Acori Tatarinowii, Pericarpium Citri Reticulatae, Rhizoma Pinelliae, and Poria are used to dry Dampness and resolve Phlegm; some herbs frequently used to activate Blood and resolve Stasis are Radix Angelicae Sinensis, Rhizoma Chuanxiong, Radix Paeoniae Rubra, Semen Persicae, Flos Carthami, Radix Cyathulae, and Sanguis Draconis. ${ }^{41,43,44}$

TCM is traditionally regarded as having few side-effects. It is therefore becoming increasingly popular with the public. Recently, many clinical trials reported that CHM had a positive effect in treating infertile women with ovulatory dysfunction. ${ }^{45-50}$ However, most of the clinical trials were based on a small sample size. As there is currently insufficient evidence about the safety and efficacy of CHM for the management of infertility with anovulation, a systematic review in this area is warranted.

\section{Methods}

Articles were retrieved from databases by electronic search. Only articles that satisfied the selection criteria were included in the meta-analysis. The articles were reviewed independently by 2 reviewers. After selection, the data were extracted by the first reviewer and verified by the second reviewer. Discrepancies were rectified while referring to the original articles.

\section{Criteria for Considering Studies for This Review}

\section{Types of studies}

Randomized controlled trials (RCTs) were included. Quasi-RCTs, non-RCTs, or randomized trials with false methods for random allocation of participants were excluded. Articles were written in either English or Chinese language.

\section{Types of participants}

Female patients, of any age or ethnic origin, who were incapable of conceiving for at least 1 year (includes primary and secondary subfertility) as a result of anovulation, were included in the study. Luteal phase defect, tubal disease, immune infertility, and unexplained infertility were excluded. Sperm analysis of the partner should be normal. The diagnostic criteria of anovulation employed in the present review were based on the American Society of Reproductive Medicine report, ${ }^{51}$ the standard drafted by the Obstetrics and Gynecology Committee of The China Association of Integrative Medicine, ${ }^{52}$ and the guiding principle issued by the Ministry of Health of the People's Republic of China. ${ }^{53}$

Anovulation was diagnosed if an individual had two or more of the following six criteria: (1) Monophasic BBT recordings lasted for more than 3 months, (2) Serum or urine progesterone levels that fell below the normal values during the midluteal phase, (3) Endometrial biopsy and histologic evaluation that demonstrated no secretory endometrial development during the late luteal phase (6 days before menstruation), (4) Transvaginal ultrasound reveal no evidence of ovulation, (5) Vaginal cytology smears showing no cyclical changes, or (6) Cervical mucus crystallization that occurred without ellipsoid.

Ideally, the diagnostic criteria of anovulation should be stated and described in the trial that was searched for this review. If the criteria were not employed in the searched trials, the stated diagnostic criteria in each individual study were evaluated by the review authors to confirm whether it met above the criteria. Trials with inconsistent diagnostic criteria were excluded. If the diagnostic criteria were not clearly stated in the trial, the primary authors were contacted for clarification. In case clarification was unavailable, these trials were also excluded.

\section{Types of Interventions}

Experimental interventions encompassed Chinese patent herbal medicine, extracts of a single herb or compound of herbs, or other individualized herbal remedies. Control interventions comprised placebo, no treatment, Western medicine, and laparoscopic surgery. 


\section{Types of Outcome Measures}

Primary outcomes include live birth rate, pregnancy rate, and ovulation rate. Secondary outcomes include the change of BBT, cervical mucus score, endometrial thickness, follicle growth, miscarriage, ectopic pregnancy and adverse events (including OHSS, luteinized unruptured follicle syndrome (LUFS), multiple pregnancy, gastrointestinal reactions, headaches, general malaise, etc.).

\section{Search Strategy}

The terms retrieved in databases were infertile, infertility, sterile, sterility, anovulation, anovulatory, ovulatory dysfunction, ovulatory disorders, ovulation failure, ovarian Stimulation, ovarian Induction, Chinese traditional, Chinese medicine, alternative medicine, Complementary Therap\$, chinese herbal, Plants, plant extract\$, herb\$. The combined search was (infertile OR infertility OR steril OR sterility) AND (anovulation OR anovulatory OR ovulatory dysfunction OR ovulatory disorders OR ovulation failure OR ovarian Stimulation OR Ovarian Induction) AND (Chinese traditional OR Chinese medicine OR alternative medicine OR Complementary Therap\$ OR chinese herbal OR Plants OR plant extract\$ OR herb\$). Chinese language databases were retrieved with a similar search strategy.

\section{Databases}

A total of eight databases were extensively searched. The Chinese electronic databases included VIP Information (19892011.3), CMCC (1994-2011.3), and CNKI (1979-2011.3). The English electronic databases included AMED (1985-2011.3), CINAHL (1982-2011.3), Cochrane Library (1993-2011.3), Embase (1996-2011.3) and MEDLINE ${ }^{\circledR}$ (1966-2011.3).

\section{Data Analysis}

The Review Manager 4.2 software developed at the Nordic Cochrane Centre was employed for data analysis. Di- chotomous data were presented as odds ratio (OR) and continuous outcomes as mean difference, both with $95 \%$ confidence intervals $(\mathrm{CI})$. Differences were considered statistically significant, and $p$-value was $<0.05$.

\section{Results}

\section{Study selection}

The initial searches identified 692 articles on treatment with TCM for infertile women with ovulatory dysfunction. On reading titles and abstracts, it was found that 626 of these articles were excluded because they were duplicates, nonclinical studies, case reports, or had study objectives different from this review. A total of 66 articles published in Chinese were retrieved for further assessment. Of these, 51 articles were excluded because they did not meet the inclusion criteria of this review. In total, 15 articles that satisfied the selection criteria were included. ${ }^{54-68}$

\section{Methodological quality of included studies}

There were 1659 participants who enrolled in 15 studies. Sample size ranged from 46 to 335 . Nine hundred and eighty-two (982) were allocated to the treatment group and 677 were allocated to the control group. All participants were women of reproductive age, but with infertility and anovulation. All clinical studies were conducted in China and reported in the Chinese language. Though the clinical trials claimed randomization in their studies, all of these trials were marked as high-risk bias due to the unclear allocation concealment, blindness, and withdrawal/dropout. All studies were classified according to the Jadad scale, ${ }^{69}$ as shown in Table 1.

\section{Study characteristics}

Periodic therapies by using different CHM were applied in four studies. Chinese patent drugs were used in six studies.

Table 1. Quality Evaluation of Included Studies

\begin{tabular}{|c|c|c|c|c|c|c|c|c|}
\hline \multirow[b]{2}{*}{ Study } & \multirow[b]{2}{*}{ Treatment $(\mathrm{N})$} & \multirow[b]{2}{*}{ Control $(\mathrm{N})$} & \multicolumn{6}{|c|}{ Quality Assessment } \\
\hline & & & Ran. & All Con & Blind & With/drop & $T S$ & JSS \\
\hline Cui et al. (2003) & 120 & 80 & A & $\mathrm{C}$ & $\mathrm{C}$ & $\mathrm{C}$ & HRB & 2 \\
\hline Huang et al. (2006) & 90 & 45 & $\mathrm{~A}$ & $\mathrm{C}$ & $\mathrm{C}$ & $\mathrm{C}$ & HRB & 2 \\
\hline Liu (2010) & 30 & 30 & A & $\mathrm{C}$ & $\mathrm{C}$ & $\mathrm{C}$ & HRB & 2 \\
\hline Huang (2007) & 32 & 20 & A & $\mathrm{C}$ & $\mathrm{C}$ & $\mathrm{C}$ & HRB & 2 \\
\hline Qiu Fen-lian et al. (2004) & 25 & 21 & B & $\mathrm{C}$ & $\mathrm{C}$ & $\mathrm{C}$ & HRB & 1 \\
\hline Pang et al. (1997) & 59 & 53 & B & $\mathrm{C}$ & $\mathrm{C}$ & $\mathrm{C}$ & HRB & 1 \\
\hline Xu (2009) & 30 & 30 & $\mathrm{~B}$ & $\mathrm{C}$ & $\mathrm{C}$ & $\mathrm{C}$ & HRB & 1 \\
\hline Qiu Ming-ying et al. (2004) & 30 & 30 & $\mathrm{~B}$ & $\mathrm{C}$ & $\mathrm{C}$ & $\mathrm{C}$ & $\mathrm{HRB}$ & 1 \\
\hline Luo et al. (2007) & 182 & 153 & $\mathrm{~B}$ & $\mathrm{C}$ & $\mathrm{C}$ & $\mathrm{C}$ & $\mathrm{HRB}$ & 1 \\
\hline Chu et al. 2006 & 60 & 60 & $\mathrm{~B}$ & $\mathrm{C}$ & $\mathrm{C}$ & $\mathrm{C}$ & HRB & 1 \\
\hline Yin et al. (2006) & 120 & 40 & $\mathrm{~B}$ & $\mathrm{C}$ & $\mathrm{C}$ & $\mathrm{C}$ & HRB & 1 \\
\hline Yin et al. (2004) & 76 & 32 & $\mathrm{~B}$ & $\mathrm{C}$ & $\mathrm{C}$ & $\mathrm{C}$ & HRB & 1 \\
\hline Xia et al. (2004) & 45 & 23 & $\mathrm{~B}$ & $\mathrm{C}$ & $\mathrm{C}$ & $\mathrm{C}$ & HRB & 1 \\
\hline $\mathrm{Fu}(2007)$ & 34 & 20 & B & C & C & C & HRB & 1 \\
\hline Huang (2002) & 49 & 40 & B & $\mathrm{C}$ & $\mathrm{C}$ & $\mathrm{C}$ & HRB & 1 \\
\hline Subtotal & 982 & 677 & & & & & & \\
\hline Total & 1659 & & & & & & & \\
\hline
\end{tabular}

Ran, randomization; All Con, allocation concealment; Blind, blindness; With/drop, withdrawal/dropout; A, adequate; B, inadequate; C, unclear; HRB, high-risk bias; TS, total score; JSS, Jadad scale score. 
Chinese herbal formulas were used in five studies. Details are listed in Table 2.

There were 13 studies used clomiphene only as the controls; 4 of them used clomiphene from $50 \mathrm{mg} / \mathrm{d}$ to $100 \mathrm{mg} / \mathrm{d}$ or $150 \mathrm{mg} / \mathrm{d}$ when no ovulation occurred in the first two cycles. For the other two studies, Qiu Ming-ying et al. (2004) used clomiphene combined with diethylstilbestrol and medroxyprogesterone as the controls, and Fu (2007) selected clomiphene combined with human chorionic gonadotrophin as the controls.

The duration of trials selected in this study varied from 3 months to 6 months, yet the duration recorded in one article was 1 month $^{67}$ while another article did not report the trial duration. ${ }^{59}$ All articles have reported the diagnostic criteria used, with all participants diagnosed with infertility and anovulation in these studies.

Pregnancy rate (per woman) was reported in all studies. Ovulation rate (per woman) was reported in 13 studies. The change of BBT was reported in five studies. Improvement of cervical mucus score was reported in two studies. Change of endometrial thickness was reported in three studies. Follicle growth was reported in six studies. Adverse events were reported in four studies. Live birth rate (per woman) was not measured by any included studies (Table 3 ).

\section{Effects and adverse events of interventions}

There were 13 trials (1545 women) that compared one of the commonly used CHM with clomiphene, and two trials (114 women) that compared CHM with combination therapy. Subgroups were set up for statistical analysis based on the type of CHM used and different combination therapy as control.

\section{Pregnancy rate}

$\mathrm{CHM}$ versus clomiphene (see Analysis 1.1). There were 13 trials (1545 women) that reported statistically significant difference between $\mathrm{CHM}$ and clomiphene $(58.50 \%$ versus $30.46 \%$; OR 3.12, 95\% CI 2.50-3.88). This meta-analysis included four trials (615 women) compared periodic therapies to clomiphene $(60.50 \%$ versus $33.20 \%$; OR 2.95 , 95\% CI 2.09 4.17); four trials (501 women) compared Chinese patent drugs to clomiphene (64.07\% versus $29.61 \%$; OR $4.20,95 \%$ CI 2.86-6.17); the other five trials (429 women) compared Chinese herbal formulas to clomiphene $(49.43 \%$ versus $27.38 \%$; OR 2.36, 95\% CI 1.55-3.61).

$\mathrm{CHM}$ versus clomiphene combination therapy (see Analysis 2.1). There were two trials (114 women) that reported no statistically significant difference between $\mathrm{CHM}$ and combination therapy (51.56\% versus $34 \%$; OR $2.12,95 \%$ CI $0.98-4.57)$. This meta-analysis included one trial (60 women) that compared CHM to clomiphene combined with diethylstilbestrol and medroxyprogesterone $(56.67 \%$ versus $33.33 \%$; OR 2.62, 95\% CI 0.92-7.46); the other trial (54 women) compared CHM to clomiphene combined with human chorionic gonadotrophin $(47.06 \%$ versus $35 \%$; OR $1.65,95 \%$ CI 0.53-5.16).

Table 2. Contents of the Formulations Used in Included Studies

Cui et al. (2003)

Huang et al. (2006)

Liu (2010)

Huang (2007)

Qiu Fen-lian

et al. (2004)

Pang et al. (1997)

$\mathrm{Xu}(2009)$

Qiu Ming-ying

et al. (2004)

Luo et al. (2007)

Chu et al. (2006)

Yin et al. (2006)

Yin et al. (2004)

Xia et al. (2004)

$\mathrm{Fu}$ (2007)

Huang (2002)
Homemade Chinese patent drug

Homemade Chinese patent drug

Periodic therapies

Herbal formula

Chinese patent drug

Herbal formula

Periodic therapies

Chinese patent drug

Periodic therapies

Homemade Chinese patent drug

Periodic therapies

Herbal formula

Herbal formula

Homemade Chinese patent drug

Herbal formula
Basic formula of zhu yun pill No. 1, which was produced by their hospital's Preparation room.

Basic formula of an kun zhong zi pill, which was produced by their hospital's Preparation room.

1. Basic formula of cu pai luan decoction No. 1 for late follicular phase from the 5th to 12th day of menstrual cycle; 2 . Basic formula of cu pai luan decoction No. 2 for ovulation phase and luteinizing phase from the 13th to 22nd day of menstrual cycle.

Basic formula of bu shen hua yu decoction.

Basic formula of tiao jing cu yun pill, which had national drug production batch number.

Basic formula of bu shen zhong zi pellet.

1. Basic formula of wu zi yan zong decoction for follicular phase; 2. Basic formula of cu pai luan decoction for ovulation phase; 3. Basic formula of $c u$ huang ti decoction for luteinizing phase.

Basic formula of gui lu bu shen pill, which had national drug production batch number.

1. Basic formula of tiao jing decoction No. 1 for follicular phase; 2. Basic formula of tiao jing decoction No. 2 for ovulation phase;

3. Basic formula of tiao jing decoction No. 3 for luteinizing phase; 4. Basic formula of tiao jing decoction No. 4 for menstrual phase.

Basic formula of er zi capsule, which was produced by their hospital's Preparation room.

1. Basic formula of bu shen tian jing decoction; 2. Basic formula of tao hong si wu decoction.

Basic formula of shuang $z i$ decoction.

Basic formula of $n v$ zhen yun yu decoction.

Basic formula of zhu yun pill, which was produced by their hospital's Preparation room.

Basic formula: The experimental formula for nourishing Kidney Yin and Blood. 
TAble 3. Characteristics of Included Studies

\begin{tabular}{|c|c|c|c|c|}
\hline Study & Intervention & Controlled intervention & Duration & Outcome measurement \\
\hline Cui et al. (2003) & $\begin{array}{l}\text { Homemade Chinese } \\
\text { patent drug }\end{array}$ & Clomiphene $50 \mathrm{mg} / \mathrm{d}$ & 6 months & $\begin{array}{l}\text { Pregnancy rate, ovulation rate, } \\
\text { BBT, adverse events }\end{array}$ \\
\hline $\begin{array}{l}\text { Huang et al. } \\
\qquad(2006)\end{array}$ & $\begin{array}{l}\text { Homemade Chinese } \\
\text { patent drug }\end{array}$ & Clomiphene $50 \mathrm{mg} / \mathrm{d}$ & 6 months & $\begin{array}{l}\text { Pregnancy rate, ovulation rate, } \\
\text { BBT, cervical mucus score, } \\
\text { follicle growth }\end{array}$ \\
\hline Liu (2010) & Periodic therapies & Clomiphene $50 \mathrm{mg} / \mathrm{d}$ & 3 months & Pregnancy rate, ovulation rate \\
\hline Huang (2007) & Herbal formula & Clomiphene $50 \mathrm{mg} / \mathrm{d}$ & 6 months & Pregnancy rate, ovulation rate \\
\hline $\begin{array}{l}\text { Qiu Fen-lian } \\
\text { et al. (2004) }\end{array}$ & Chinese patent drug & Clomiphene $50-100 \mathrm{mg} / \mathrm{d}$ & 3 months & $\begin{array}{l}\text { Pregnancy rate, ovulation rate, } \\
\text { adverse events }\end{array}$ \\
\hline Pang et al. (1997) & Herbal formula & Clomiphene & Not mentioned & Pregnancy rate \\
\hline Xu (2009) & Periodic therapies & Clomiphene $50 \mathrm{mg} / \mathrm{d}$ & 3 months & Pregnancy rate, ovulation rate \\
\hline $\begin{array}{l}\text { Qiu Ming-ying } \\
\text { et al. (2004) }\end{array}$ & Chinese patent drug & $\begin{array}{l}\text { Clomiphene } 50 \mathrm{mg} / \mathrm{d} \\
\text { Diethylstilbestrol } 0.25 \mathrm{mg} / \mathrm{d} \\
\text { Medroxyprogesterone } 1 \mathrm{mg} / \mathrm{d}\end{array}$ & 3-6 months & $\begin{array}{l}\text { Pregnancy rate, ovulation rate, } \\
\text { endometrial thickness, } \\
\text { follicle growth }\end{array}$ \\
\hline Luo et al. (2007) & Periodic therapies & Clomiphene $50 \mathrm{mg} / \mathrm{d}$ & 3 months & $\begin{array}{l}\text { Pregnancy rate, ovulation rate, } \\
\text { BBT, adverse events }\end{array}$ \\
\hline chu et al. 2006 & $\begin{array}{l}\text { Homemade Chinese } \\
\text { patent drug }\end{array}$ & Clomiphene $50 \mathrm{mg} / \mathrm{d}$ & 3 months & $\begin{array}{l}\text { Pregnancy rate, ovulation rate, } \\
\text { BBT, follicle growth, adverse } \\
\text { events }\end{array}$ \\
\hline Yin et al. (2006) & Periodic therapies & Clomiphene $50 \mathrm{mg} / \mathrm{d}$ & 6-9 months & $\begin{array}{l}\text { Pregnancy rate, ovulation rate, } \\
\text { follicle growth }\end{array}$ \\
\hline Yin et al. (2004) & Herbal formula & Clomiphene $50 \mathrm{mg} / \mathrm{d}$ & 3-6 months & $\begin{array}{l}\text { Pregnancy rate, ovulation rate, } \\
\text { follicle growth }\end{array}$ \\
\hline Xia et al. (2004) & Herbal formula & Clomiphene $50-100 \mathrm{mg} / \mathrm{d}$ & 3 months & $\begin{array}{l}\text { Pregnancy rate, ovulation rate, } \\
\text { endometrial thickness, follicle } \\
\text { growth, adverse events }\end{array}$ \\
\hline $\mathrm{Fu}(2007)$ & $\begin{array}{l}\text { Homemade Chinese } \\
\text { patent drug }\end{array}$ & $\begin{array}{l}\text { Clomiphene } 50 \mathrm{mg} / \mathrm{d} \\
\text { HCG 5000-10,000 U }\end{array}$ & 1 month & $\begin{array}{l}\text { Pregnancy rate, BBT, cervical } \\
\text { mucus score, endometrial } \\
\text { thickness }\end{array}$ \\
\hline Huang (2002) & Herbal formula & Clomiphene $50-100 \mathrm{mg} / \mathrm{d}$ & 6 months & Pregnancy rate, ovulation rate \\
\hline
\end{tabular}

BBT, basal body temperature; HCG, human chorionic gonadotropin.

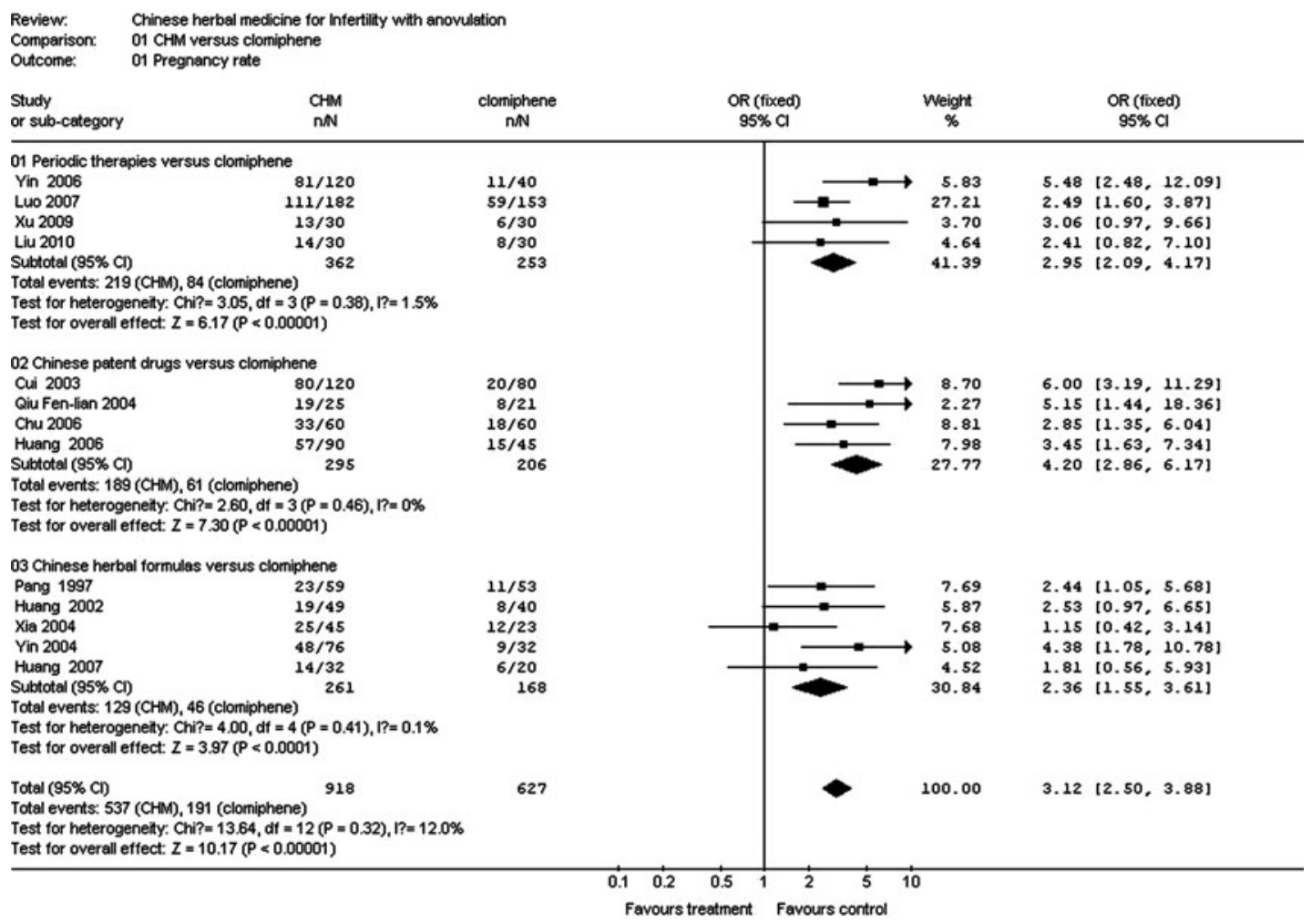

ANALYSIS 1.1. Comparison 1. Chinese herbal medicine (CHM) versus clomiphene, outcome 1: pregnancy rate (per woman). 


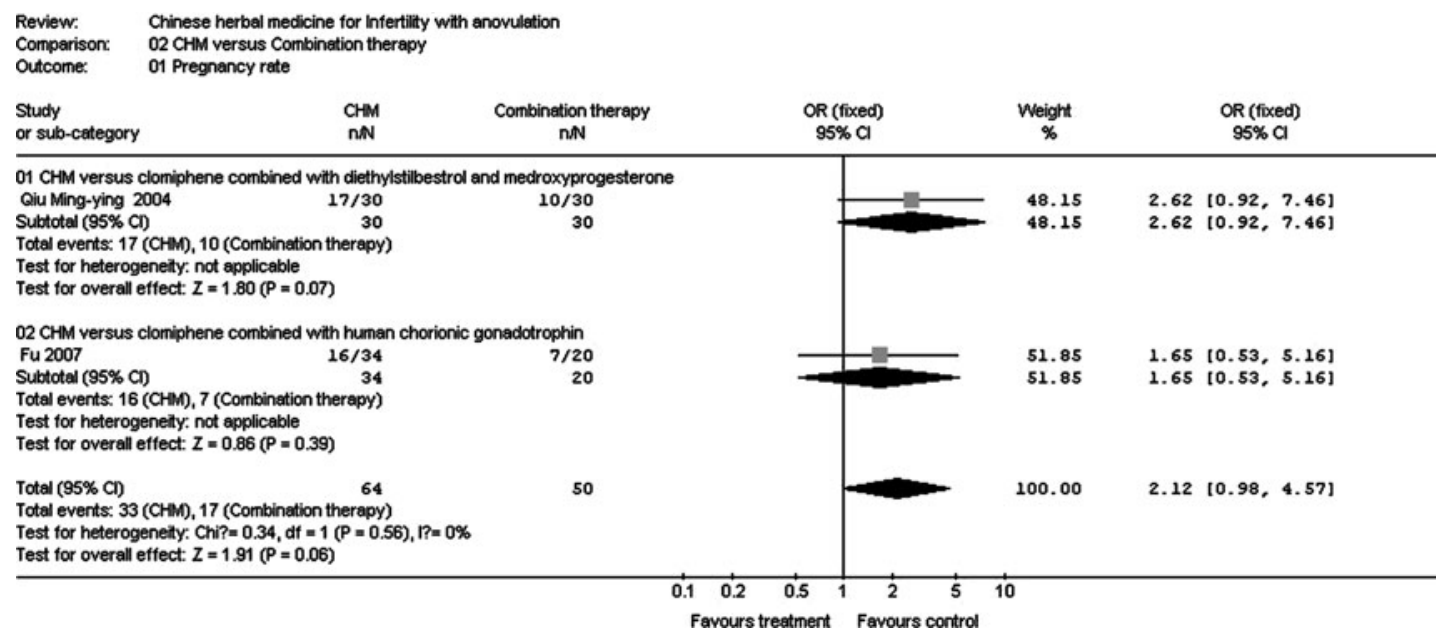

ANALYSIS 2.1. Comparison 2. Chinese herbal medicine (CHM) versus clomiphene combination therapy, outcome 1 pregnancy rate.

\section{Ovulation rate}

$\mathrm{CHM}$ versus clomiphene (see Analysis 1.2). There were 11 trials (1365 women) that compared CHM with clomiphene. Four trials (615 women) reported no statistically significant difference between periodic therapies and clomiphene $(73.20 \%$ versus $62.45 \%$; OR $1.7,95 \%$ CI $0.87-3.32$ ). Four (4) trials (501 women) reported no statistically significant difference between Chinese patent drugs and clomiphene $(79.32 \%$ versus $69.90 \%$; OR $1.53,95 \%$ CI $0.72-3.26$ ). The other three trials (249 women) reported no statistically significant difference be- tween Chinese herbal formulas and clomiphene (84.71\% versus $81.52 \%$; OR $1.32,95 \%$ CI $0.64-2.71)$.

Overall, meta-analysis of 11 trials (1365 women) showed that there was a statistically significant difference between CHM and clomiphene (78.81\% versus $69.69 \%$; OR $1.55,95 \%$ CI 1.06-2.25).

$\mathrm{CHM}$ versus clomiphene combination therapy (see Analysis 2.2). One (1) trial (60 women) compared CHM to clomiphene combined with diethylstilbestrol and medroxy-

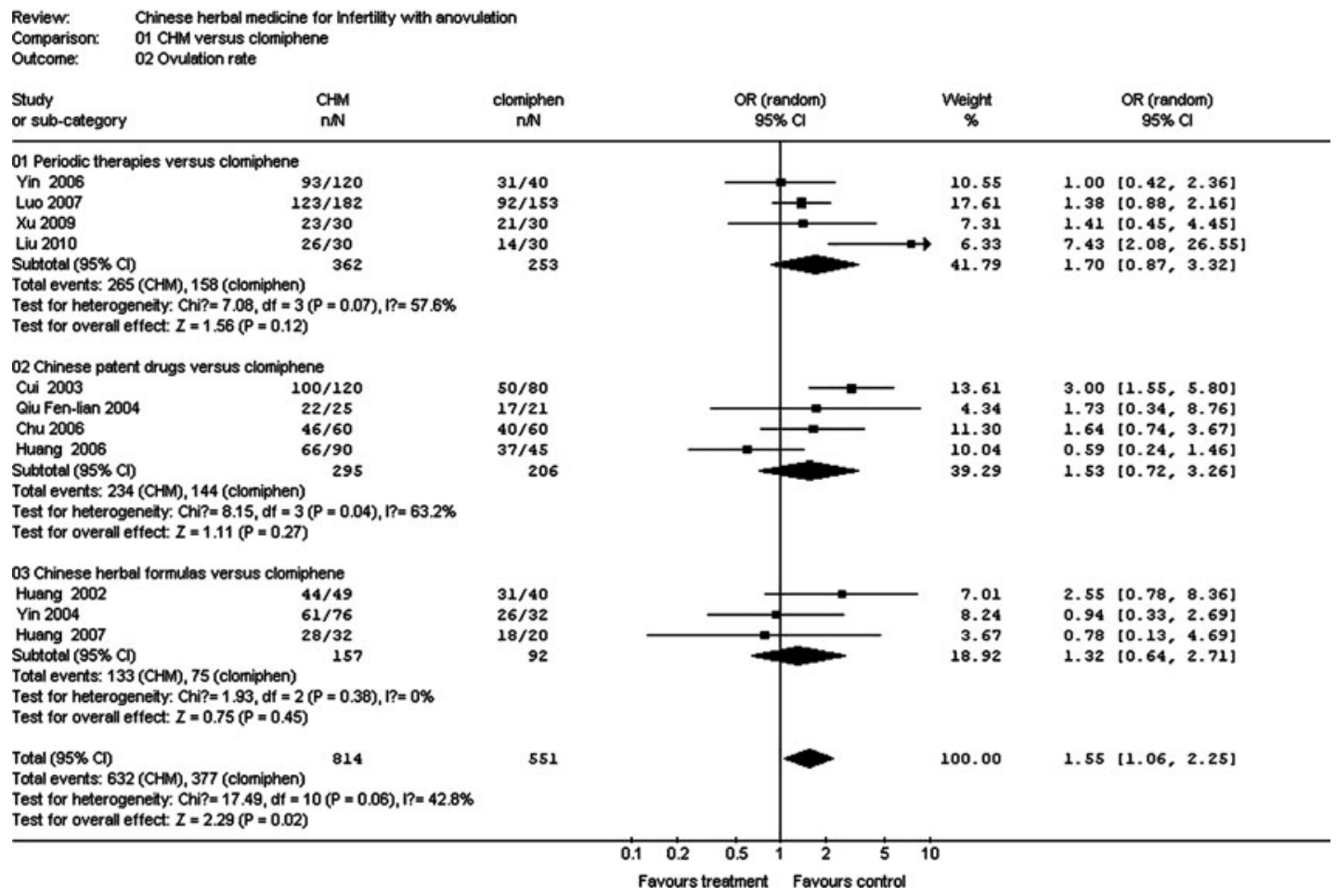

ANALYSIS 1.2. Comparison 1. Chinese herbal medicine (CHM) versus clomiphene, outcome 2: ovulation rate (per woman). 


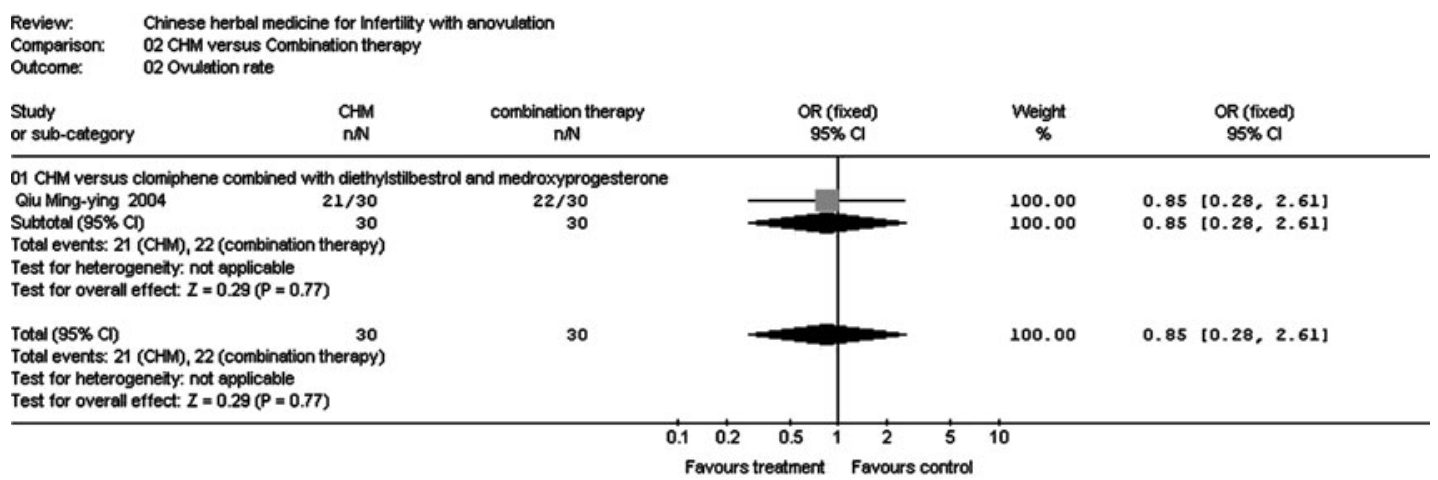

ANALYSIS 2.2. Comparison 2. Chinese herbal medicine (CHM) versus clomiphene combination therapy, outcome 2 ovulation rate (per woman).

progesterone. The difference shown was not statistically significant (70\% versus $73.33 \%$; OR $0.85,95 \%$ CI $0.28-2.61$ ).

\section{BBT becoming biphasic}

CHM versus clomiphene (see Analysis 1.3). Three (3) trials (596 women) reported this outcome, one as typical biphasic $\mathrm{BBT}^{62}$ and another two as atypical and typical biphasic BBT. ${ }^{55,63}$ We used the typical biphasic BBT as outcome for final comparison.

Meta-analysis of three trials (596 women) showed that there was no statistically significant difference between CHM and clomiphene $(65.78 \%$ versus $64.59 \%$; OR $1.06,95 \%$ CI $0.73-1.53)$

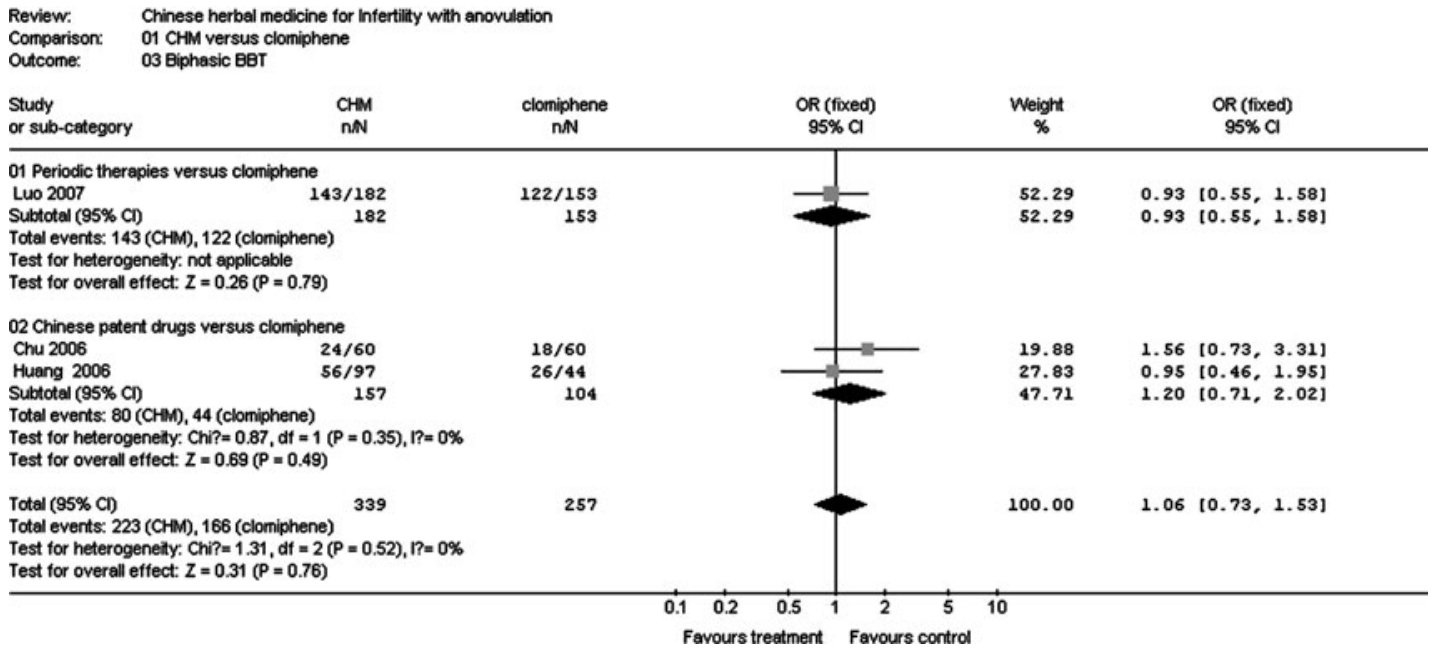

ANALYSIS 1.3. Comparison 1. Chinese herbal medicine (CHM) versus clomiphene, outcome 3: basal body temperature becoming biphasic.

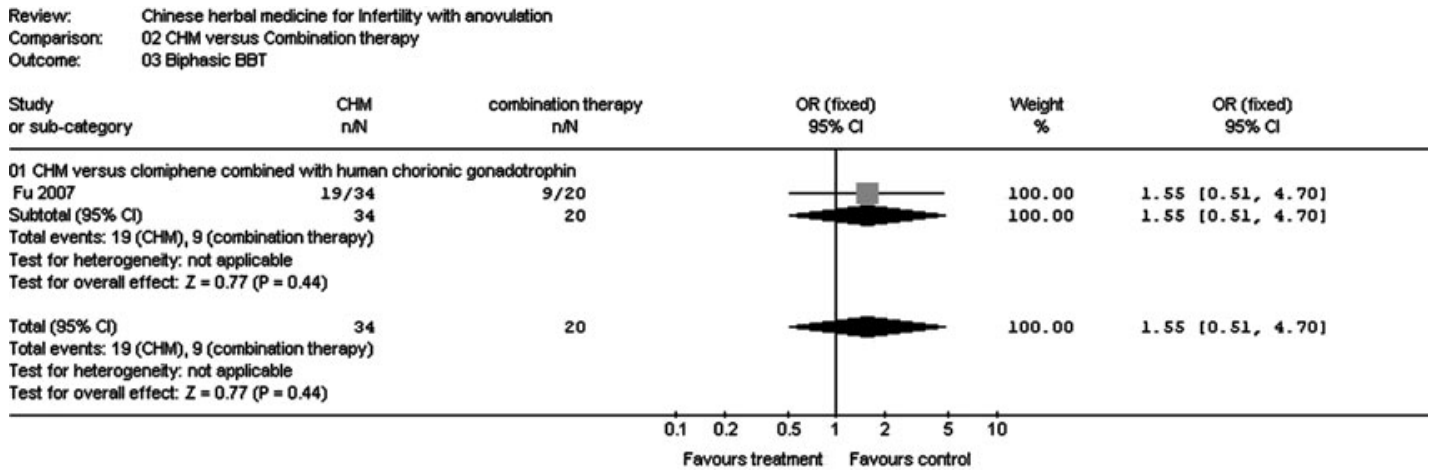

ANALYSIS 2.3. Comparison 2. Chinese herbal medicine (CHM) versus clomiphene combination therapy, outcome 3 basal body temperature becoming biphasic. 


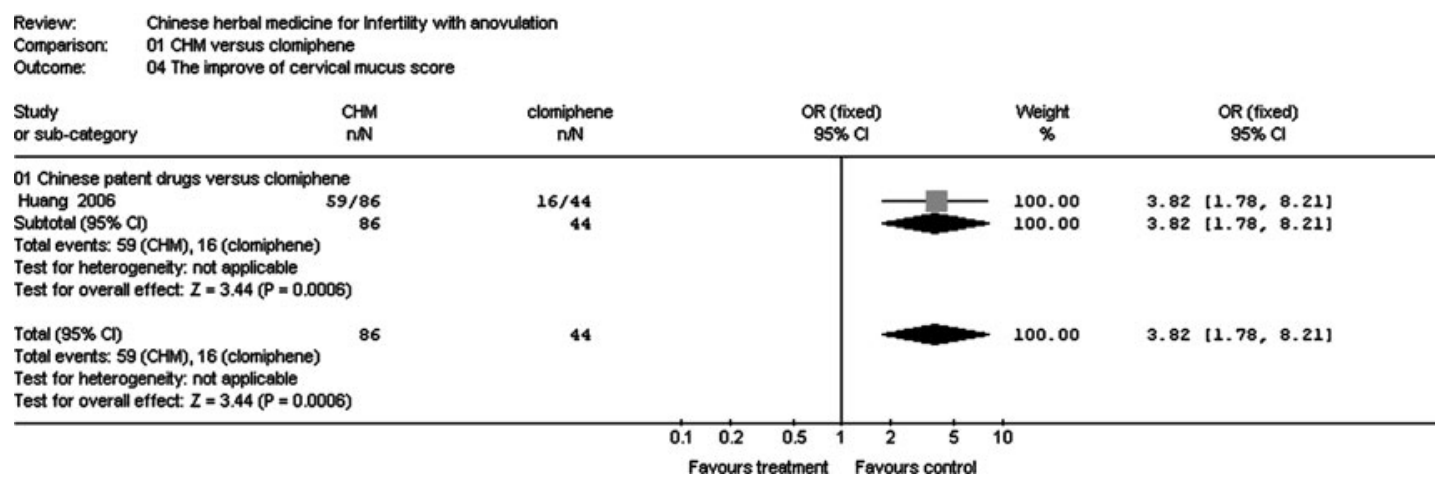

ANALYSIS 1.4. Comparison 1. Chinese herbal medicine (CHM) versus clomiphene, outcome 4: the improvement of cervical mucus score.

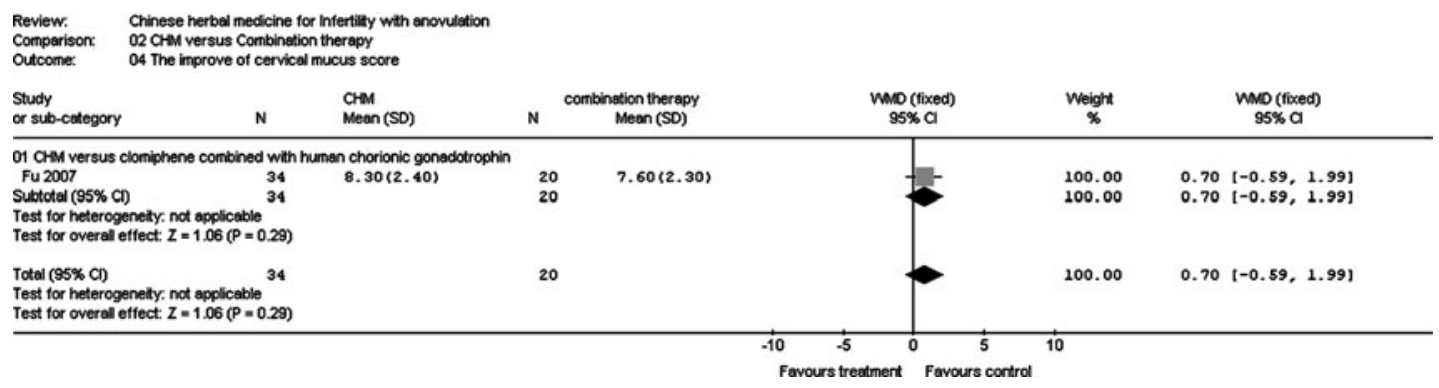

ANALYSIS 2.4. Comparison 2. Chinese herbal medicine (CHM) versus clomiphene combination therapy, outcome 4 the improvement of cervical mucus score.

$\mathrm{CHM}$ versus clomiphene combination therapy (see Analysis 2.3). One (1) trial (54 women) compared CHM to clomiphene combined with chorionic gonadotrophin. The difference showed there was not a statistically significant difference (55.88\% versus $45 \%$; OR 1.55 , $95 \%$ CI $0.51-4.70$ ).

\section{Improvement of cervical mucus score}

$\mathrm{CHM}$ versus clomiphene (see Analysis 1.4). One (1) trial (130 women) compared CHM against clomiphene. ${ }^{55}$ Results showed that CHM was significantly more effective for improving cervical mucus score than clomiphene was $(68.60 \%$ versus $36.36 \%$; OR 3.82, 95\% CI 1.78-8.21).

$\mathrm{CHM}$ versus clomiphene combination therapy (see Analysis 2.4). One (1) trial (54 women) compared CHM against combination therapy. ${ }^{67}$ Results showed that CHM was not significantly more effective for improving cervical mucus score than clomiphene combined with human chorionic gonadotrophin (weighted mean difference [WMD] 0.70, 95\% CI -0.59-1.99).

\section{Change of endometrial thickness}

$\mathrm{CHM}$ versus clomiphene. One (1) trial, ${ }^{66}$ which only reported the average thickness of two groups (no standard deviation), showed a significant increase in thickness after treatment in the treated group, but no significant difference in the control group.

CHM versus clomiphene combination therapy (see Analysis 2.5). There were two trials (114 women) that reported no statistically significant difference between CHM and combination therapy (WMD 0.39, 95\% CI -0.15-0.94).

\section{Follicle growth}

$\mathrm{CHM}$ versus clomiphene (see Analysis 1.6). There were five trials that compared CHM with clomiphene. One (1) trial $^{66}$ only reported the average size of two groups. There were no standard deviations provided, so it was not included in meta-analysis.

Four trials (523 women) reported that this outcome was included in meta-analysis, one trial (160 women) used periodic therapies, ${ }^{64}$ another two trials (255 women) used Chinese patent drugs, ${ }^{55,63}$ and another trial (108 women) used Chinese herbal formulas. ${ }^{64}$

Meta-analysis of four trials (523 women) showed that there was no statistically significant difference between CHM and clomiphene (WMD - 0.37 95\% CI -2.12-1.38).

$\mathrm{CHM}$ versus clomiphene combination therapy (see Analysis 2.6). One (1) trial (60 women) compared CHM to clomiphene combined with diethylstilbestrol and medroxyprogesterone. The difference showed no statistically significant difference (WMD - 0.75 95\% CI - 1.66-0.16).

\section{Miscarriage rate (see Analysis 1.7)}

There were three trials (938 women in trial, 307 women were pregnant) that reported a statistically significant difference between CHM and clomiphene $(6.01 \%$ versus $27.47 \%$; OR 0.2 , 95\% CI $0.10-0.41$ ). This meta-analysis included one trial (170 women were pregnant) that compared 


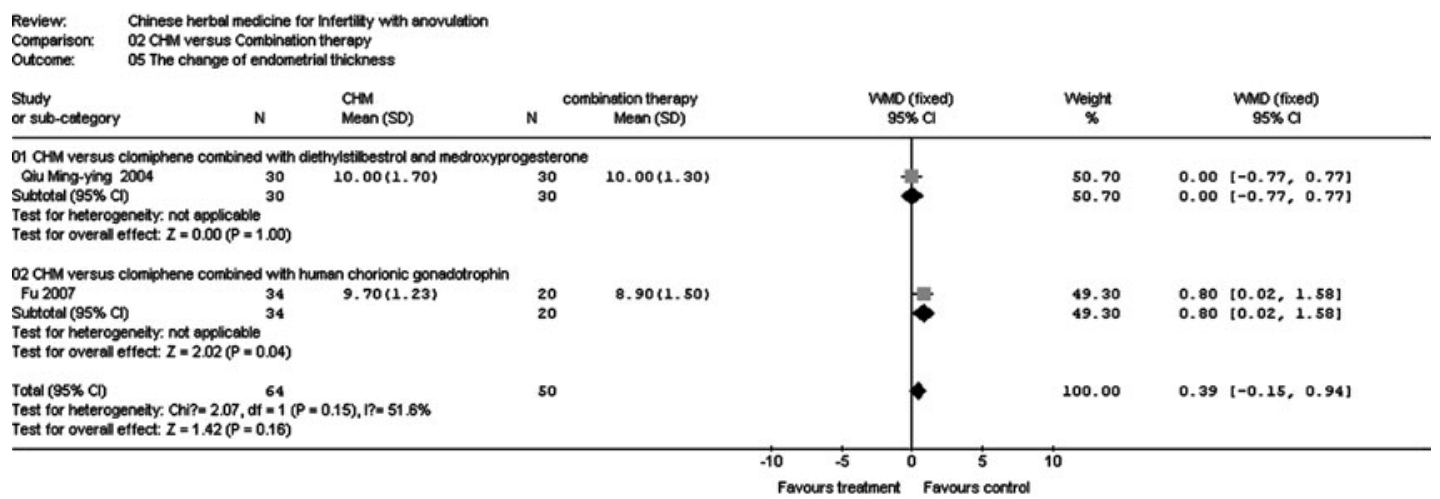

ANALYSIS 2.5. Comparison 2. Chinese herbal medicine (CHM) versus clomiphene combination therapy, outcome 5 the change of endometrial thickness.

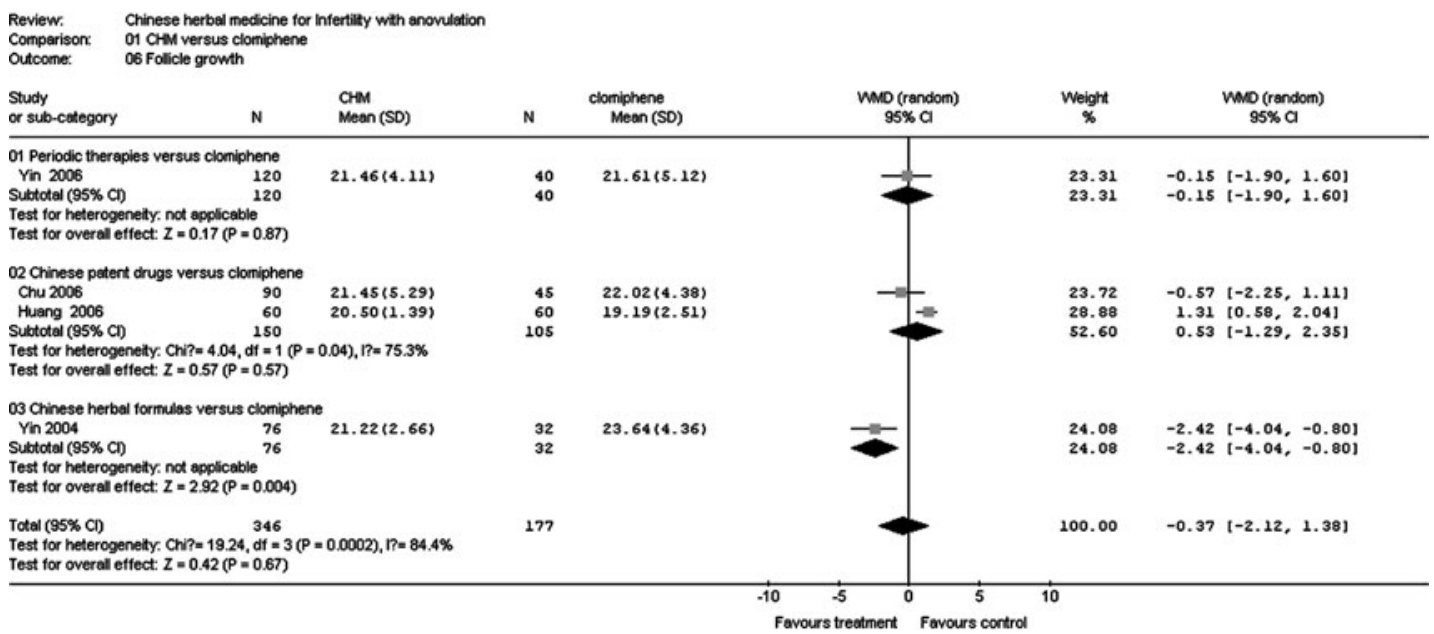

ANALYSIS 1.6. Comparison 1. Chinese herbal medicine (CHM) versus clomiphene, outcome 6: follicle growth.

periodic therapies to clomiphene $(9.91 \%$ versus $32.2 \%$; OR $0.23,95 \%$ CI $0.10-0.53$ ); one trial (100 women were pregnant) compared Chinese patent drugs to clomiphene $(0 \%$ versus 20\%; OR 0.02 , 95\% CI $0.00-0.44$ ); the other trial (37 women were pregnant) compared Chinese herbal formulas to clomiphene ( $8 \%$ versus $16.67 \%$; OR $0.43,95 \% \mathrm{CI}$ 0.05-3.44).

\section{Ectopic pregnancy (see Analysis 1.8)}

One (1) trial (335 women in trial, 170 women were pregnant) reported no statistically significant difference between
CHM (periodic therapies) and clomiphene (1.8\% versus 8.47\%; OR 0.2, 95\% CI 0.4-1.05).

\section{Adverse events}

OHSS (see Analysis 1.9). Only one study (335 women) reported OHSS. There was no statistically significant difference between CHM (periodic therapies) and clomiphene for OHSS (0\% versus $6.54 \%$; OR $0.28,95 \%$ CI $0.01-6.89) .{ }^{64}$

LUFS (see Analysis 1.10). Only one study (335 women) reported LUFS. There was a statistically significant difference

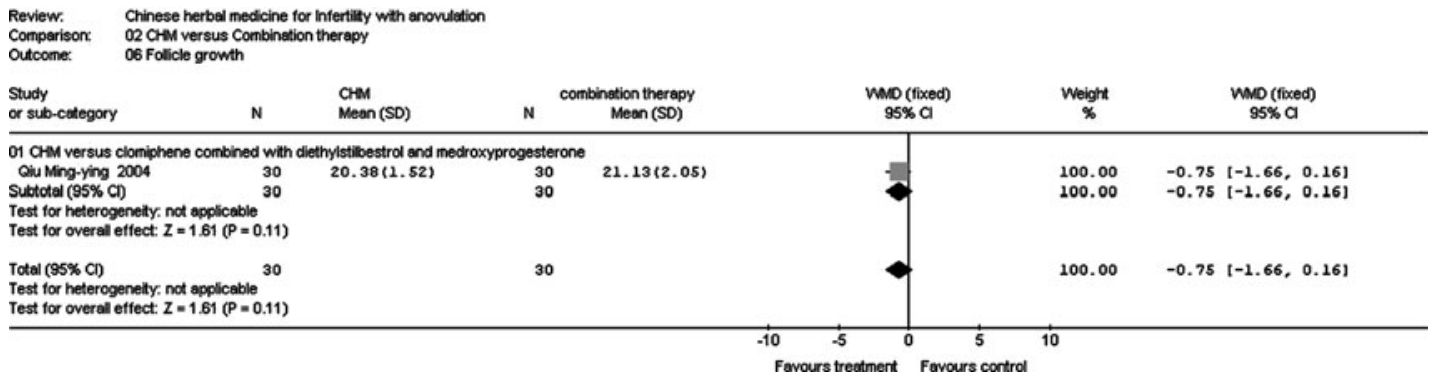

ANALYSIS 2.6. Comparison 2. Chinese herbal medicine (CHM) versus clomiphene combination therapy, outcome 6 follicle growth. 


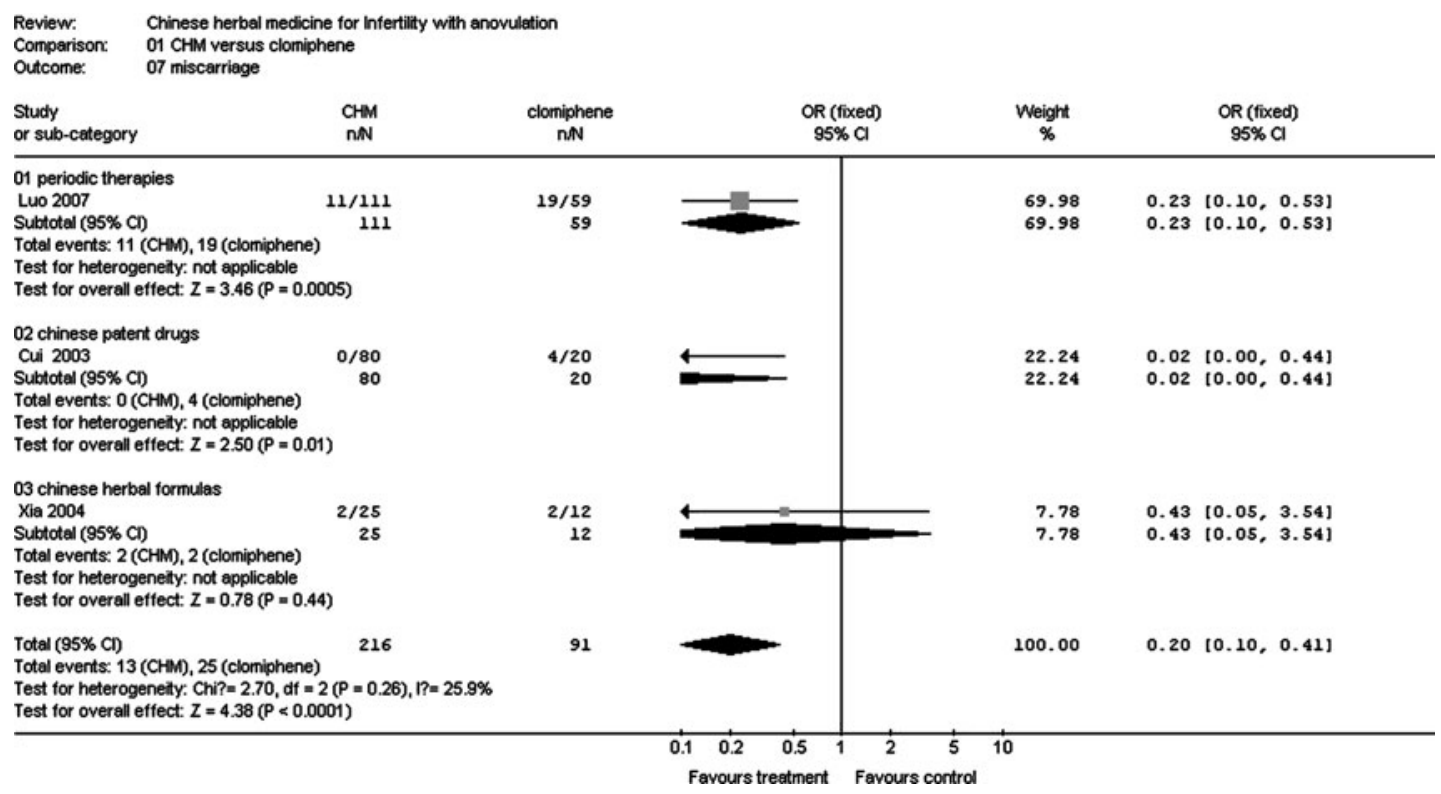

ANALYSIS 1.7. Comparison 1. Chinese herbal medicine (CHM) versus clomiphene, outcome 7: miscarriage rate.

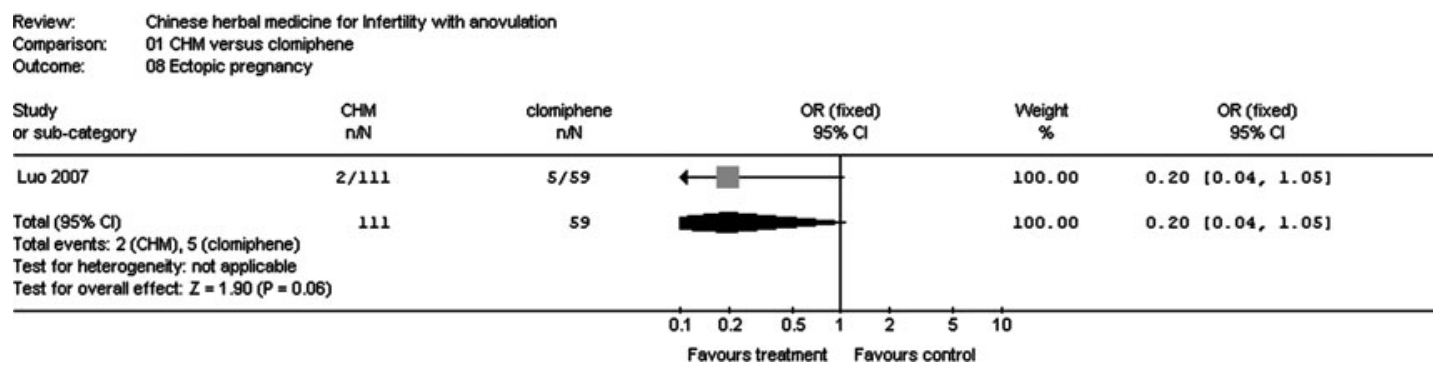

ANALYSIS 1.8. Comparison 1. Chinese herbal medicine (CHM) versus clomiphene, outcome 8: ectopic pregnancy.

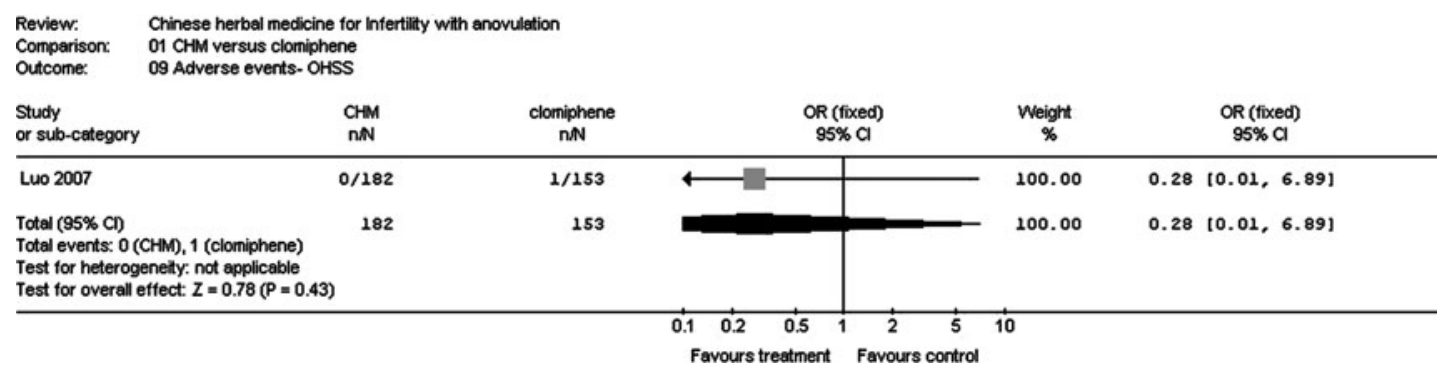

ANALYSIS 1.9. Comparison 1. Chinese herbal medicine (CHM) versus clomiphene, outcome 9: adverse events, ovarian hyperstimulation syndrome (OHSS Chinese herbal medicine).

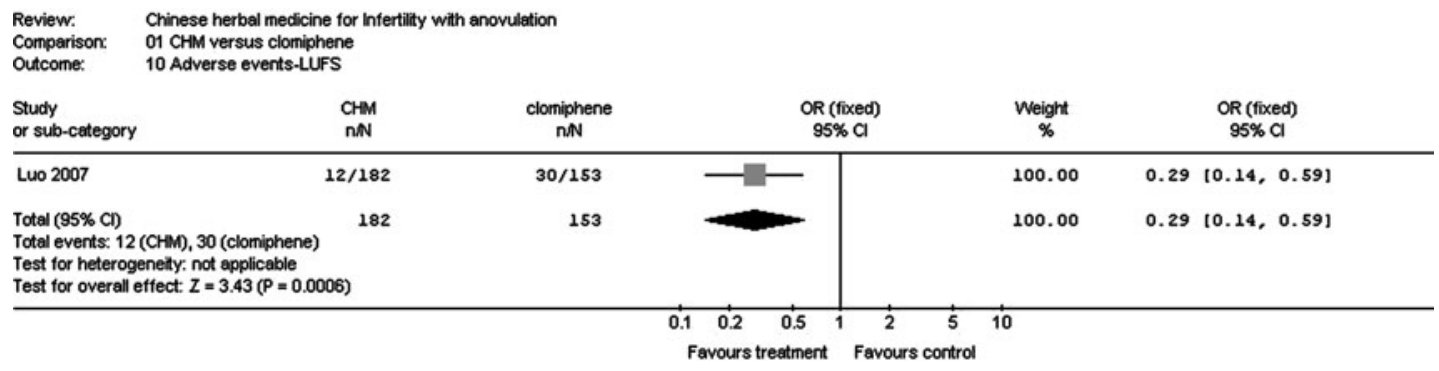

ANALYSIS 1.10. Comparison 1. Chinese herbal medicine (CHM) versus clomiphene, outcome 10: adverse events, luteinized unruptured follicle syndrome (LUFS). 


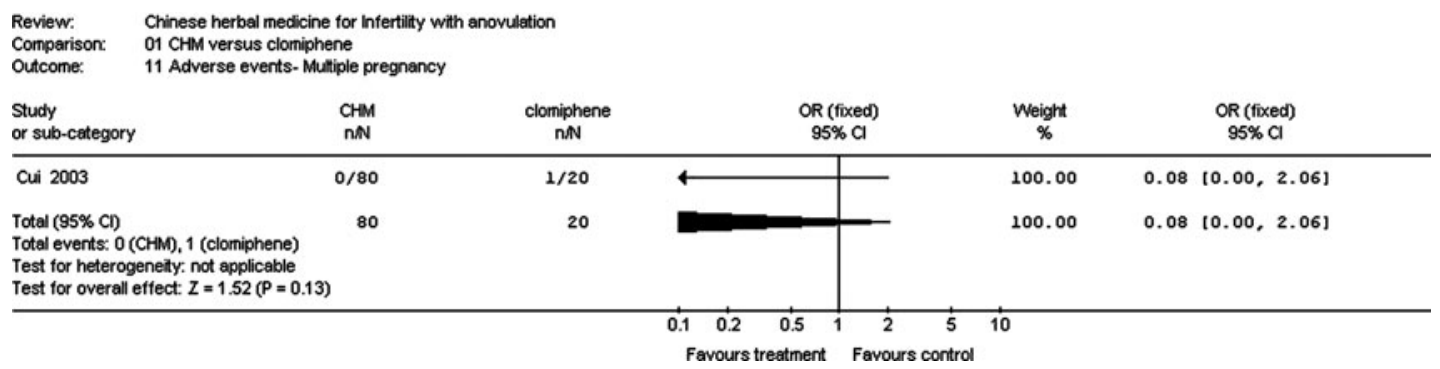

ANALYSIS 1.11. Comparison 1. Chinese herbal medicine (CHM) versus clomiphene, outcome 11 adverse events: multiple pregnancy.

between CHM (periodic therapies) and clomiphene for LUFS (6.59\% versus $19.61 \%$; OR $0.29,95 \%$ CI $0.14-0.59){ }^{64}$

Multiple pregnancy (see Analysis 1.11). Only one study (200 women in trial, 100 women were pregnant) reported multiple pregnancy. There was no statistically significant difference between CHM (periodic therapies) and clomiphene for multiple pregnancy $(0 \%$ versus $4 \%$; OR $0.08,95 \%$ CI 0.00-2.06). ${ }^{54}$

Other no serious adverse events (see Analysis 1.12). Two (2) trials (166 women) reported no serious adverse events (including tiredness, dizziness, headache, palpitations, nausea, vomiting, diarrhea, dry mouth, drowsiness, abnormal uterine bleeding, urticaria, atopic dermatitis, breast pain, and weight gain) in either the treated or control groups. Meta-analysis of two trials (166 women) showed that there was no statistically significant difference between CHM (Chinese patent drugs) and clomiphene (4.71\% versus $29.63 \%$; OR $0.09,95 \%$ CI $0.00-1.67) .{ }^{58,63}$

\section{Live birth rate}

This outcome index was not reported in any of the included studies.

\section{Discussion}

The aim of this review was to assess the effectiveness of $\mathrm{CHM}$ and its safety for the treatment of anovulation and infertility in women. CHM has been used to treat conditions in female patients for more than 2000 years. However, there is a dearth data on the administration of $\mathrm{CHM}$ in a rigorous scientific context.
The review included 15 trials, which furnished promising evidence in the form of RCTs for the use of CHM in increasing pregnancy rate and ovulation rate, improving cervical mucus score, reducing miscarriage rate in the treatment of infertility with anovulation, but did not present other evidence of any other effects. Live birth rate, as the most important outcome for infertile patients, is unclear for CHM in treating women with anovulation and infertility. No significant adverse effects were identified for the use of CHM from the included studies in this review.

\section{Impact of Individualized Approaches of CHM}

Traditional treatment with CHM needs to be tailored according to different patterns, according to a description of symptoms and signs attributed to different syndromes. Moreover, a pattern has individual variations and changes over time with different stages of disease. In CHM, treatment differs according to the identified and diagnosed syndrome.

Six of 15 included trials considered an inclusion criterion in relation to the TCM diagnostic syndrome (all were the syndrome of deficiency of the Kidney), and another 9 trials considered only Western diagnostic criteria. In other words, the minority of included trials paid special attention to the role of differentiated syndrome defined in TCM diagnosis for infertility, because the syndrome of infertility in TCM informs the selection of treatment formulation. The syndrome of Deficiency of the Kidney was the common diagnostic classification of infertility in TCM diagnosis. ${ }^{70-73}$ This was in agreement with the majority of published diagnostic protocols in TCM. The selection of CHM used in the trials in this review was mostly influenced by the TCM diagnostic process.

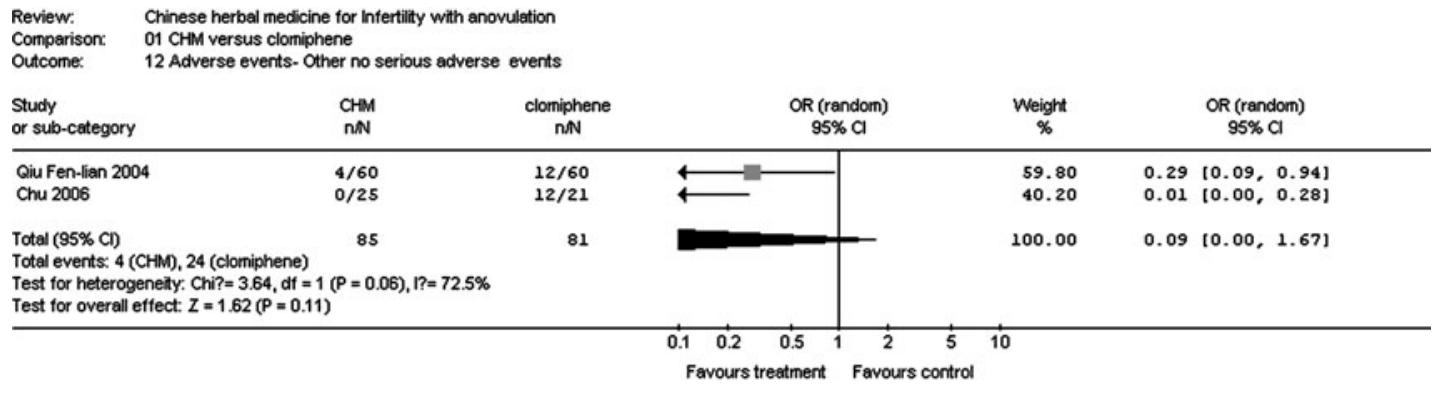

ANALYSIS 1.12. Comparison 1. Chinese herbal medicine (CHM) versus clomiphene, outcome 12 adverse events: other not serious adverse events. 
Some trials used periodic therapies in accordance with the individual syndromes and different stages. Some trials used Chinese patent drugs and Chinese herbal formulas across the whole period of clinical intervention, either because one diagnostic syndrome of infertility was selected for this specific patent drug or formula, or simply because no consideration was given to the possible variation of diagnostic syndromes. Therefore, this review was unable to provide an explicit answer as to whether periodic therapies were more beneficial than Chinese patent drugs or Chinese herbal formulas in the treatment of infertility with anovulation.

\section{Adverse Effects}

Although one study reported a statistically significant difference between CHM and clomiphene for LUFS, the safety of CHM in clinical practice was not addressed adequately in the reviewed trials. The measurement and report of adverse effects were poor; most trials neglected the fact that herbs are not risk free. Only three trials mentioned some adverse effects and detailed the number of incidents, not only in the treated groups but also in the control groups. This allowed appropriate data to be extracted for meta-analysis. Generally, the reviewed trials reported that use of CHM for treatment of infertility with anovulation was safe when compared with ovulation-induction agents (clomiphene). Adverse events including LUFS, OHSS and multiple pregnancy have been reported in only one study, but without reporting of the severity. Other adverse events occasionally induced by CHM are not reported comprehensively, such as impairment of liver and kidney, and allergy. More trials that monitor possible adverse effects are needed.

\section{Quality of the Evidence}

Jadad scores of the 15 RCTs were 1-2, which means that the methodological quality of these RCTs was of a poor caliber. Except for randomization methods, no trial clearly described their methods of allocation concealment, blindness, or withdrawal/dropout.

Another weakness was the small sample size. Half of the trials had less than 100 participants. The small size reduces the likelihood of detecting the effect of an intervention in a

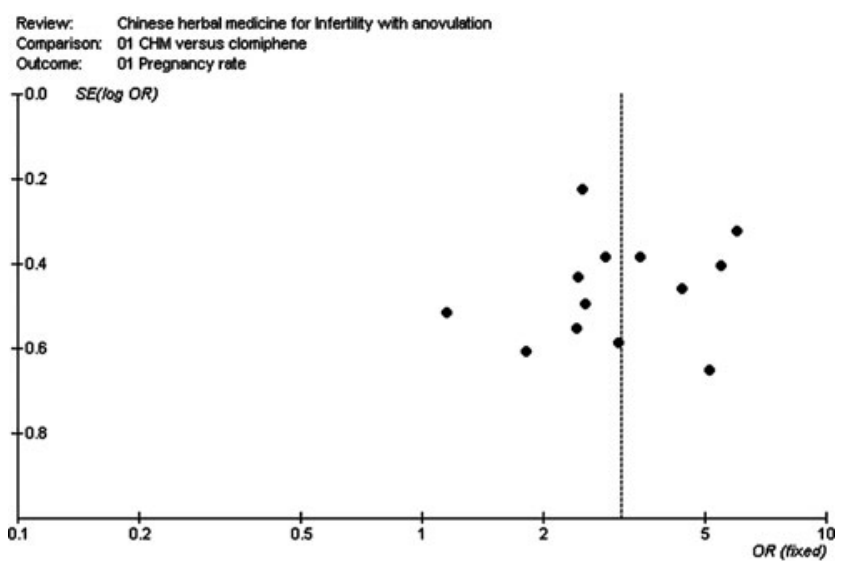

FIG. 1. Funnel plot of trials comparing Chinese herbal medicine (CHM) with clomiphene for the outcome of pregnancy rate. SE, standard error; $\mathrm{OR}$, odds ratio. single study, but given the similarities between studies, it makes meta-analysis of the data particularly valuable.

The lack of placebo trials affects the results, since typically a medicine's efficacy is established with placebo trials before comparisons are made with other drugs. In addition, the lack of blinding in these trials might affect the results as it may give skewed results, if participants were aware of their treatment.

A possible publication bias has not been excluded in this review, because the majority of trials reported positive effects of CHM in the treatment of infertility. Funnel plots were used to investigate the possibility of publication bias, for the trials comparing CHM with clomiphene. For the outcomes of pregnancy rate, the funnel plots appeared roughly symmetrical, indicating less chances of publication bias in this group of trials (Fig. 1).

Overall, this review has found that an attempt toward evidence-based TCM practice has been made. However, good design of multicentered, randomized, parallel-controlled and blinding trials is needed before undertaking further studies, with the aim of providing better evidencebased medicine evidence.

\section{Acknowledgments}

This research was supported by Shanghai Leading Academic Discipline Project, Project Number: S30303.

\section{Disclosure Statement}

No financial conflicts exist.

\section{References}

1. Kumar A, Ghadir S, Eskandari N, DeCherney AH. Infertility. In: DeCherney AH, Nathan L, Goodwin TM, Laufer $\mathrm{N}$, eds. Current Diagnosis and Treatment. Obstetrics \& Gynecology. New York: McGraw Hill, 2007.

2. Juul S, Karmaus W, Olsen J. Regional differences in waiting time to pregnancy: Pregnancy-based surveys from Denmark, France, Germany, Italy and Sweden. The European Infertility and Subfecundity Study Group. Hum Reprod 1999;14:1250-1254.

3. Olsen J, Kuppers-Chinnow M, Spinelli A. Seeking medical help for subfecundity: A study based upon surveys in five European countries. Fertil Steril 1996;66:95-100.

4. Rutstein SO, Shah IH. Infecundity, Infertility, and Childlessness in Developing Countries. DHS Comparative Reports No. 9. Calverton, MD: ORC Macro and the World Health Organization, 2004.

5. Mosher WD, Pratt WF. Fecundity and infertility in the United States: Incidence and trends. Fertil Steril 1991;56: 192-193.

6. Zhang L-Z. Clinical Reproductive Endocrinology and Infertility. China, Beijing: Science Press, 2001:445.

7. Cedars M, Jaffe RB. Patient information page from the hormone foundation: Infertility and women. J Clin Endocrinol Metab 2005;90:4.

8. Luciano AA, Peluso J, Koch EI, et al. Temporal relationship and reliability of clinical, hormonal, and ultrasonographic indices of ovulation in infertile women. Obstet Gynecol 1990;75:412-416.

9. Wathen NC, Perry L, Lilford RJ, Chard T. Interpretation of single progesterone measurement in diagnosis of anovula- 
tion and defective luteal phase: Observations on analysis of the normal range. Br Med J 1984;288:7-9.

10. Noyes RW, Hertig AW, Rock J. Dating the endometrial biopsy. Fertil Steril 1950;1:3.

11. de Crespigny LC, O'Herlihy C, Robinson HP. Ultrasonic observation of the mechanism of human ovulation. Am J Obstet Gynecol 1981;139:636-639.

12. Hull MG, Glazener CM, Kelly NJ, et al. Population study of causes, treatment, and outcome of infertility. Br Med J (Clin Res Ed) 1985;291:1693-1697.

13. Baird DT. Ovulation induction: Current status and future prospects of gonadotrophin therapy. In: Adashi EY, Leung PC, eds. The Ovary. New York: Raven Press, 1993:529-544.

14. Gaware VM, Parjane SK, Merekar Abhijit N, et al. Female infertility and its treatment by alternative medicine: A review. J Chem Pharm Res 2009;1:148-162.

15. MacGregor AH, Johnson JE, Bunde CA. Further clinical experience with clomiphene citrate. Fertil Steril 1968;19:616-622.

16. Hughes E, Collins J, Vandekerckhove P. Clomiphene citrate for ovulation induction in women with oligo-amenorrhoea. Cochrane Database Syst Rev 2000;2:CD000056.

17. Platteau P, Nyboe Andersen A, Balen A, et al. for the Menopur Ovulation Induction (MOI) Study Group. Similar ovulation rates, but different follicular development with highly purified menotrophin compared with recombinant FSH in WHO Group II anovulatory infertility: A randomized controlled study. Hum Reprod 2006;21:1798-1804.

18. Balen A, Platteau $\mathrm{P}$, Nyboe Andersen A, et al. for the Bravelle Ovulation Induction (BOI) Study Group. Highly purified FSH is as efficacious as recombinant FSH for ovulation induction in women with WHO Group II anovulatory infertility: A randomized controlled non-inferiority trial. Hum Reprod 2007;22:1816-1823.

19. Lee VC, Ledger W. Aromatase inhibitors for ovulation induction and ovarian stimulation. Clin Endocrinol (Oxf) 2011;74:537-46.

20. The Thessaloniki ESHRE/ASRM-Sponsored PCOS Consensus Workshop Group. Consensus on infertility treatment related to polycystic ovary syndrome. Hum Reprod 2008;23: 462-477.

21. Derman SG, Adashi EY. Adverse effects of fertility drugs. Drug Saf 1994;11:408-421.

22. Pelinck MJ, Hoek A, Simons AH, Heineman MJ. Efficacy of natural cycle IVF: A review of the literature. Hum Reprod Update 2002;82:129-139.

23. Kashyap S, Moher D, Fung MF, Rosenwaks Z . Assisted reproductive technology and the incidence of ovarian cancer: A meta-analysis. Obstet Gynecol 2004;103:785-794.

24. Ayhan A, Salman MC, Celik H, et al. Association between fertility drugs and gynecologic cancers, breast cancer, and childhood cancers. Acta Obstet Gynecol Scand 2004;83: 1104-1111.

25. Brinton L. Long-term effects of ovulation-stimulating drugs on cancer risk. Reprod Biomed Online 2007;15:38-44.

26. Petignat $\mathrm{P}$, Vassilakos $\mathrm{P}$, Campana A. Are fertility drugs a risk factor for persistent trophoblastic tumour? Hum Reprod 2002;17:1610-1615.

27. Gauthier E, Paoletti X, Clavel-Chapelon F; E3N group. Breast cancer risk associated with being treated for infertility: Results from the French E3N cohort study. Hum Reprod 2004;19:2216-2221.

28. Jensen A, Sharif H, Frederiksen K, Kjaer SK. Use of fertility drugs and risk of ovarian cancer: Danish Population Based Cohort Study. Am J Epidemiol 2009;170:1408-1414.
29. Lerner-Geva L, Rabinovici J, Lunenfeld B. Ovarian stimulation: Is there a long-term risk for ovarian, breast and endometrial cancer? Womens Health (Lond Engl) 2010;6:831-839.

30. Corchia C, Mastroiacovo P, Lanni R, et al. What proportion of multiple births are due to ovulation induction? A registerbased study in Italy. Am J Public Health 1996;86:851-854.

31. Kurinczuk JJ, Hansen M, Bower C. The risk of birth defects in children born after assisted reproductive technologies. Curr Opin Obstet Gynecol 2004;16:201-209.

32. Hansen M, Bower C, Milne E, et al. Assisted reproductive technologies and the risk of birth defects: A systematic review. Hum Reprod 2005;20:328-38.

33. Olson CK, Keppler-Noreuil KM, Romitti PA, et al. In vitro fertilization is associated with an increase in major birth defects. Fertil Steril 2005;84:1308-1315.

34. Zhou J, Qu F. Treating gynaecological disorders with traditional Chinese medicine: A review. Afr J Tradit Complement Altern Med 2009;6:494-517.

35. Huang ST, Chen AP. Traditional Chinese medicine and infertility. Curr Opin Obstet Gynecol 2008,20:211-215.

36. Yan F, Zhang JH, Li RF, Song LP. Integrative Chinese and western medicine approach in treating 64 patients with anovular infertility. Zhongguo Zhong Xi Yi Jie He Za Zhi 2003;9:60-62.

37. MacLennan AH, Wilson DH, Taylor AW. Prevalence and cost of alternative medicine in Australia. Lancet 1996;347: 569-573.

38. Eisenberg D, David RB, Ettner SL, et al. Trends in alternative medicine use in the United States; 1990-1997. JAMA 1998; 280:1569-1575.

39. Ernst E, White AR. The BBC survey of complementary medicine use in the UK. Complement Ther Med 2000;8: 32-36.

40. Chen G, Shi Y. The development of Chinese Medicine treatment in anovulatory infertility. J Jiangxi Univ Trad Chin Med 2009;21:84-86.

41. Liu Y, Zhou H. Progress of Traditional Chinese Medicine in treating anovulatory infertility. J Liaoning Univ Trad Chin Med 2010;12:280-281.

42. Feng G. Discussion on application mechanism of treating ovulation barriers with Xiaoyao San. Clin J Chin Med 2010; 2:13-14.

43. Han Y, Wang H, Qiao J, et al. Recent developments of Chinese Medicine and Western medicine for treatment of anovulatory infertility. J Liaoning Univ Trad Chin Med 2011;13:5-7.

44. Mao E, Zhou H. Summary on TCM treating ovulation dysfunction infertility. J Liaoning Univ Trad Chin Med 2009; 11:72-74.

45. Mo X, Li D, Pu Y, et al. Clinical studies on the mechanism of acupuncture stimulation of ovulation. J Trad Chin Med 1993;13:115-119.

46. Liu JX, Liu MR, Song T. Clinical and experimental study on the ovulation promoting effect of yanging decoction. Zhongguo Zhong Xi Yi Jie He Za Zhi 2001;21:94-98.

47. Yin X, Jiang M, Zuo X. Clinical research of "Shuangzi decoction" in treating anovulatory infertility in 76 cases. Shanghai J Trad Chin Med 2004;38:39-41.

48. Lian F, Wang L, Zhang JW. Effect of erzhi tiangui recipe on ovarian reactivity in elderly sterile women. Zhongguo Zhong Xi Yi Jie He Za Zhi 2006;26:685-688.

49. Li S, Li Y, Chang F. Clinical observation of Yishen Zhuyun granule in treating 120 cases with infertility due to ovulatory dysfunction. J Nanjing Univ Trad Chin Med 2009;25:31-34. 
50. Liu D. A comparative study on ovulation decoction in treating ovulation failure barrenness. Clin J Chin Med 2010;2:40-41.

51. Practice Committee of the American Society for Reproductive Medicine. Optimal evaluation of the infertile female. Fertil Steril 2006;86(5 suppl 1):S264-S267.

52. Obstetrics and Gynecology Committee of The China Association of Integrative Medicine the Third Conference. Female Infertility Integrative Medicine standard. Chin J Integr Med 1991;11:378.

53. The Ministry of Health of the People's Republic of China. The Guiding Principles of Chinese Medicine New Drugs Clinical Research (First Series). China, Beijing: China Medical Science Press 1993:276-279.

54. Cui NN, Li GJ. 120 cases of Zhuyun No.1 Tang on ovulatory dysfunction infertility. Shanxi Zhong Yi 2003;24:963-964.

55. Huang TH, Luo L, Ma J, et al. Clinical and experimental research on Ankun Zhongzi Wan on follicle development and ovulation. Gansu Zhong Yi 2006;19:45-47.

56. Liu DF. A comparative study on ovulation decoction in treating ovulation failure barrenness. Clin J Chin Med 2010;15:40-41.

57. Huang YL. 32 cases of Bushen Huayu Tang on ovulatory dysfunction infertility. Shizhen Guo Yi Guo Yao 2007;18: 2237.

58. Qiu LF, Wang J. Clinical observation of TiaoJing Cuyun Wan on ovulatory dysfunction infertility. Guoji Yi Yao Wei Sheng Dao Bao 2004;10:66-67.

59. Pang BZ, Zhao HY. Prospective study of 59 cases on nourish kidney and ovulation. Shanxi Zhong Yi 1997;18:488.

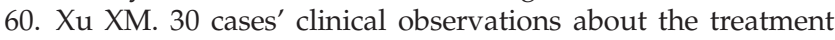
cycle of Bushen Huoxue medicine on ovulatory dysfunction infertility. Chin Health Care 2009;17:789-790.

61. Qiu MY, Luo Q, Zhu LY, et al. Clinical observations of Guilu Bushen capsule on ovulation induction. Liaoning Zhong Yi Za Zhi 2004;31:479-480.

62. Luo ZJ, Zhan J, Wu YY. 182 cases of Tiaozhou Zhongyao Fang on ovulatory dysfunction infertility. Zhongyi Za Zhi 2007; 48:432-433.

63. Chu YX, Wang RJ. 60 cases of Erzi capsule on ovulatory dysfunction infertility. Zhongyi Yan Jiu 2006;19:27-29.

64. Yin XQ, Zuo XM. Clinical research about 160 cases of Bushen Tianjing method on ovulatory dysfunction infertility. Zhonghua Lin Chuang Yi Xue Za Zhi 2006;7:43-44.
65. Yin XQ, Jiang MS, Zuo XM. Clinical research about 76 cases of Shuangzi Tnag on ovulatory dysfunction infertility. Shanghai Zhong Yi Yao Za Zhi 2004;38:39-41.

66. Xia YW, Cai LX, Zhang SC, et al. Therapeutic effect of Chinese herbal medicines for nourishing blood and reinforcing Shen in treating patients with anovulatory sterility of Shen-deficiency type and its influence on the hemodynamics in ovarian and uterine arteries. Zhongguo Zhong $\mathrm{Xi}_{\mathrm{i}}$ Yi Jie He Za Zhi 2004;24:299-302.

67. Fu J. Clinical analysis about Chinese herbal medicines of Zhongyao Zhuyun Wan on ovulatory dysfunction infertility. Henan Zhong Yi Xue Yuan Xue Bao 2007;22:51-52.

68. Huang J. Clinical observation of nourish kidney on ovulatory dysfunction infertility. Zhejiang Zhong Xi Yi Jie He Za Zhi 2002;12:577-578.

69. Jadad AR, Moore RA, Carroll D, et al. Assessing the quality of reports of randomized clinical trials: Is blinding necessary? Control Clin Trials 1996;17:1-12.

70. Zhang M. Chinese mechanism and integrative medicine for ovulatory infertility research progress. Gansu J Trad Chin Med 2007;20:70-72.

71. Gao X, Gao Y, Feng J. Clinical research of Traditional Chinese Medicine for ovulatory infertility. J Changchun Univ Trad Chin Med 2007;23:107-108.

72. Chen G, Shi Y. The development of Chinese Medicine treatment in anovulatory infertility. J Jiangxi Univ Trad Chin Med 2009;21:84-86.

73. Xu L, Luo S. Pathogenesis of ovulation disorders. Chin J Infor TCM 2003;10:4.

Address correspondence to: Ting-ting Zhang, PhD YueYang Hospital of Integrative Chinese \& Western Medicine Affiliated with Shanghai University of Traditional Chinese Medicine 110 Gan He Road Hongkou District Shang Hai China

E-mail: tingting185@yahoo.com.cn 